\title{
Convex Hodge Decomposition and Regularization of Image Flows
}

\author{
Jing Yuan, Christoph Schnörr, Gabriele Steidl ${ }^{\ddagger}$
}

April 14, 2008

\begin{abstract}
The total variation (TV) measure is a key concept in the field of variational image analysis. In this paper, we focus on vector-valued data and derive from the Hodge decomposition of image flows a definition of TV regularization for vector-valued data that extends the standard componentwise definition in a natural way. We show that our approach leads to a convex decomposition of arbitrary vector fields, providing a richer decomposition into piecewise harmonic fields rather than piecewise constant ones, and motion texture. Furthermore, our regularizer provides a measure for motion boundaries of piecewise harmonic image flows in the same way, as the TV measure does for contours of scalar-valued piecewise constant images.
\end{abstract}

\section{Introduction}

The total variation (TV) measure has been introduced in image processing by Rudin, Osher and Fatemi [1] in connection with image denoising. Let $B V(\Omega)$ be the space of functions of bounded variation and TV the corresponding norm which will be precisely defined in Section 2. Minimizing over $B V(\Omega)$ the convex functional

$$
\frac{1}{2}\|u-d\|_{L_{2}(\Omega)}^{2}+\lambda \operatorname{TV}(u), \quad \lambda>0
$$

for given image data $d$ leads to an edge-preserving nonlinear smoothing process that effectively removes noise and small-scale spatial patterns from $d$.

*Dept. Math.\& Comp. Sci., University of Heidelberg, yuanjing@math . uni-heidelberg.de

${ }^{\dagger}$ Dept. Math.\& Comp. Sci., University of Heidelberg, schnoerr@math.uni-heidelberg.de

${ }^{\ddagger}$ Dept. Math.\& Comp. Sci., University of Mannheim, steidl@math. uni-mannheim.de 
Starting with the work of Meyer [2], the more general viewpoint of image decomposition has been adopted, see also [3] and references therein. The solution $\bar{u}$ of the basic model (1) leads to a decomposition

$$
d=\bar{u}+\bar{v}
$$

of the given image data $d$ into a piecewise constant image structure $\bar{u}$ and oscillatory patterns and noise contained in $\bar{v}$.

Another key property of the TV measure is due to its geometric interpretation via the coarea formula [4]: $\mathrm{TV}(u)$ equals the length of level lines of $u$, summed up over the range of $u$ (contrast). As a consequence, $\operatorname{TV}(u)$ measures the length of contours of piecewise constant images, hence implements the regularization term of the Mumford-Shah variational approach to segmentation. This fact has been explored for image inpainting [5] and more recently also for image segmentation, in order to replace non-convex variational approaches $[6,7]$ by convex ones that can be globally optimized $[8,9]$.

A natural issue concerns the application of the TV measure to vector-valued data $u=$ $\left(u_{1}, u_{2}\right)^{\mathrm{T}}$. Usually, definitions are applied which, for sufficiently regular $u$, have the form [10]

$$
\operatorname{TV}(u):=\int_{\Omega} \sqrt{\left|\nabla u_{1}\right|^{2}+\left|\nabla u_{2}\right|^{2}} d x
$$

The Helmholtz decomposition of $u$ into its basic components, divergence and curl, suggests an alternative:

$$
R(u):=\int_{\Omega} \sqrt{(\operatorname{div} u)^{2}+(\operatorname{curl} u)^{2}} d x .
$$

This viewpoint has been used recently in [11] for decomposing image flows. However, a geometric interpretation based on convex analysis and its connection to the definitions (3) and (1) has not been given. We will show below that $R$

- is a mathematically more natural definition extending TV,

- decomposes flows into a richer "structural" component - analogous to $\bar{u}$ in the scalar case (2) - comprising piecewise harmonic flows rather than piecewise constant flows.

The regularizer $R$ in (4) is also relevant for the processing of directional vector fields $d$ with $\|d(x)\|_{2}=1$ a.e. in connection with other important applications besides image processing $[12,13,14]$. In fact, applying the standard TV measure (3) to the angular representation

$$
\frac{d}{\|d\|_{2}}=(\cos \theta, \sin \theta)^{\top}
$$

yields

$$
\|\nabla \theta\|_{2}=\sqrt{\left(\operatorname{div}\left(\frac{d}{\|d\|_{2}}\right)\right)^{2}+\left(\operatorname{curl}\left(\frac{d}{\|d\|_{2}}\right)\right)^{2}} .
$$

In previous work, nonlinear systems of PDEs defined on the sphere and sophisticated numerical schemes were applied for the regularization of directional fields [12, 13, 14], and a very efficient algorithm was recently devised in [14]. 
This paper, elaborates the convex representation of the term (4). Besides enabling a range of convex programming approaches for algorithm design, our approach contributes a definition of motion texture and highlights motion discontinuities to which a convex functional is sensitive.

Organization We briefly recall the basic Rudin-Osher-Fatemi (ROF) model in Section 2. In Section 3, we consider orthogonal decompositions of linear spaces of images and vector fields which provides the basis for extending the convex decomposition underlying the ROF model to vector fields in Section 4. This leads to a proper definition of the regularizer (4) in Section 5. In Section 6, we examine its behaviour at motion boundaries. A basic convex programming approach is provided in Section 7, but the design of really efficient schemes is beyond the scope of this work. Numerical experiments illustrate and validate our approach in Section 8. We conclude and indicate directions of further research in Section 9.

Notation Throughout this paper, let $\Omega \subset \mathbb{R}^{2}$ be an open, bounded and simply-connected domain with Lipschitz-continuous boundary $\partial \Omega$. Then we use the following notation for inner products of scalar fields

$$
\langle f, g\rangle_{\Omega}:=\int_{\Omega} f g d x
$$

and of vector fields

$$
\langle u, v\rangle:=u \cdot v, \quad\langle u, v\rangle_{\Omega}:=\int_{\Omega} u \cdot v d x .
$$

The scalar-valued curl operator for vector fields $v=\left(v_{1}, v_{2}\right)^{\mathrm{T}}$ is defined by

$$
\operatorname{curl} v:=\frac{\partial v_{2}}{\partial x_{1}}-\frac{\partial v_{1}}{\partial x_{2}}
$$

while the vector-valued curl operator for scalar fields $\phi$ is the rotated gradient

$$
\nabla^{\perp} \phi:=\left(\frac{\partial \phi}{\partial x_{2}},-\frac{\partial \phi}{\partial x_{1}}\right)^{\mathrm{T}}
$$

\section{The Rudin-Osher-Fatemi (ROF) Model}

The total variation of a scalar function $u$ is defined as

$$
\operatorname{TV}(u):=\sup _{\substack{\|p\|_{\infty} \leq 1 \\ p \in\left(C_{0}^{1}(\Omega)\right)^{2}}}\langle u, \operatorname{div} p\rangle_{\Omega},
$$

where $\|p\|_{\infty}:=\sup _{x \in \Omega} \sqrt{p_{1}^{2}(x)+p_{2}^{2}(x)}$. Based on this definition, the convex variational image denoising approach (1) can be transformed into its dual formulation $\bar{u}=d-\operatorname{div} \bar{p}$, where $\bar{p}$ is the minimizer of

$$
\inf _{p \in C_{\lambda}}\|\operatorname{div} p-d\|_{\Omega}^{2}
$$


where $C_{\lambda}$ is the closed convex set

$$
C_{\lambda}=\left\{v=\operatorname{div} p:\|p\|_{\infty} \leq \lambda, p \in\left(C_{0}^{1}(\Omega)\right)^{2}\right\}
$$

see [15]. Denoting the orthogonal projector onto $C_{\lambda}$ by $\Pi_{C_{\lambda}}$, this results in a decomposition of the scalar image data $d$ into

$$
d=\bar{u}+\bar{v}=\bar{u}+\operatorname{div} \bar{p}=\bar{u}+\Pi_{C_{\lambda}} d
$$

where $\bar{u}$ provides the piecewise smooth component, i.e., the large-scale structural parts of the image signal $d$, and $\operatorname{div} \bar{p}$ comprises the corresponding small-scale structure, i.e., texture and noise. Accordingly, this is called the structure-texture decomposition of image $d$.

For $d$ with mean zero, the smallest value of $\lambda$ such that the solution $\bar{u}$ of (1) becomes zero, equals the G-norm of $d$ (cf. [2]) which measures the size of the small-scale oscillating component (noise, texture) of the image function.

\section{Orthogonal Decomposition of Images and Flows}

In this section, we first provide an orthogonal decomposition of scalar-valued functions. Next, we summarize orthogonal decompositions of vector fields, the Helmholtz decomposition and, as an extension thereof, the Hodge decomposition.

The difference between orthogonal image decomposition and the convex decomposition of the previous section will provide the guideline for generalizing the convex decomposition approach to vector fields in the subsequent section.

\subsection{Orthogonal Image Decomposition}

Any scalar-valued function $d \in L^{2}(\Omega)$ can be decomposed as

$$
d=c+\operatorname{div} p,\left.\quad p\right|_{\partial \Omega}=0
$$

with a constant image $c$. Since $\int_{\Omega} \operatorname{div} p d x=0$, due to the boundary condition on $p$, the constant function $c$ is just the mean value $\frac{1}{|\Omega|} \int_{\Omega} d d x$, and we have also the orthogonality of the two components $\langle c, \operatorname{div} p\rangle_{\Omega}=0$.

As in (8), the function $d$ is decomposed into two components, a smooth component $c$ and a remaining component of small-scale structure. The difference between (8) and (9) is that the latter projects onto special convex sets, namely orthogonal subspaces. This leads to a constant "smooth" component $c$ in (9), as opposed to $u$ in (8) that tends to be piecewise constant. Of course, if $\lambda$ is large enough, then $u$ in (8) becomes a constant as well. 


\subsection{Orthogonal Decomposition of Image Flows}

Beside the Sobolev spaces $H^{1}(\Omega)$ and $H_{0}^{1}(\Omega)$ for scalar-valued functions and their componentwise analogues $\left(H^{1}(\Omega)\right)^{2}$ and $\left(H_{0}^{1}(\Omega)\right)^{2}$ for vector-valued functions, see [4], we need the special function spaces involving the div and curl of a vector field

$$
\begin{aligned}
H(\operatorname{div} ; \Omega) & =\left\{v \in L^{2}(\Omega)^{2} \mid \operatorname{div} v \in L^{2}(\Omega)\right\}, \\
H(\operatorname{curl} ; \Omega) & =\left\{v \in L^{2}(\Omega)^{2} \mid \operatorname{curl} v \in L^{2}(\Omega)\right\} .
\end{aligned}
$$

With the inner products

$$
\begin{aligned}
\langle u, v\rangle_{\operatorname{div} ; \Omega} & =\langle u, v\rangle_{\Omega}+\langle\operatorname{div} u, \operatorname{div} v\rangle_{\Omega}, \\
\langle u, v\rangle_{\operatorname{curl} ; \Omega} & =\langle u, v\rangle_{\Omega}+\langle\operatorname{curl} u, \operatorname{curl} v\rangle_{\Omega},
\end{aligned}
$$

these spaces become Hilbert spaces [16]. Next we recall the following Helmholtz decompositions.

Theorem 3.1 ([17]). The orthogonal decompositions

$$
\begin{aligned}
& L^{2}(\Omega)^{2}=\nabla H^{1}(\Omega) \oplus \nabla^{\perp} H_{0}^{1}(\Omega), \\
& L^{2}(\Omega)^{2}=\nabla H_{0}^{1}(\Omega) \oplus \nabla^{\perp} H^{1}(\Omega),
\end{aligned}
$$

into gradients and curls hold true. The gradients and curls of $H^{1}(\Omega)$ are characterized by

$$
\begin{aligned}
\nabla H^{1}(\Omega) & =\{v \in H(\operatorname{curl} ; \Omega) \mid \operatorname{curl} v=0\}, \\
\nabla^{\perp} H^{1}(\Omega) & =\{v \in H(\operatorname{div} ; \Omega) \mid \operatorname{div} v=0\} .
\end{aligned}
$$

We refine the decompositions (10) by splitting up the first factor in (10a) and the second factor in (10b), respectively.

Theorem 3.2 (Orthogonal Hodge Decomposition). The space $L^{2}(\Omega)^{2}$ can be orthogonally decomposed as

$$
L^{2}(\Omega)^{2}=\mathcal{H} \oplus \nabla H_{0}^{1}(\Omega) \oplus \nabla^{\perp} H_{0}^{1}(\Omega),
$$

where the space of harmonic vector fields $\mathcal{H}$ is given by

$$
\mathcal{H}:=\{v \in H(\operatorname{div} ; \Omega) \cap H(\operatorname{curl} ; \Omega) \mid \operatorname{div} v=\operatorname{curl} v=0\}
$$

Proof. Due to the inclusions $\nabla H_{0}^{1}(\Omega) \subset \nabla H^{1}(\Omega)$ and $\nabla^{\perp} H_{0}^{1}(\Omega) \subset \nabla^{\perp} H^{1}(\Omega)$, the space $\mathcal{H}$ is by virtue of (10) given by

$$
\mathcal{H}=\left(\nabla H_{0}^{1}(\Omega) \oplus \nabla^{\perp} H_{0}^{1}(\Omega)\right)^{\perp}=\left(\nabla H_{0}^{1}(\Omega)\right)^{\perp} \cap\left(\nabla^{\perp} H_{0}^{1}(\Omega)\right)^{\perp}=\nabla^{\perp} H^{1}(\Omega) \cap \nabla H^{1}(\Omega) .
$$

Inserting (11) yields the assertion. 


\section{Convex Hodge Decomposition of Flows}

Based on the previous discussion, natural questions arise:

1. Because the orthogonal decomposition of scalar fields (9) is clearly inferior to the convex decomposition (8) in that the structural component $\bar{u}$ models more general functions, what is the convex counterpart of the orthogonal decomposition of vector fields (12)?

2. What is the natural generalization of the variational denoising approach (1) to vector fields?

We will address the first question next and the second question in the following section. Given a vector field $d \in L^{2}(\Omega)^{2}$, we consider the convex Hodge decomposition

$$
d=\bar{u}+\bar{v}=\bar{u}+\nabla \bar{\psi}+\nabla^{\perp} \bar{\phi}=\bar{u}+\Pi_{S_{\lambda}} d,
$$

into a piecewise-smooth component $\bar{u}$ and a non-smooth component given by the orthogonal projection $\Pi_{S_{\lambda}} d$ of $d$ onto the convex set

$$
S_{\lambda}:=\left\{v=\nabla \psi+\nabla^{\perp} \phi: \sqrt{\psi(x)^{2}+\phi(x)^{2}} \leq \lambda,(\psi, \phi) \in\left(H_{0}^{1}(\Omega)\right)^{2}\right\},
$$

Notice the structural similarity between (8) and (14). Likewise, as $\bar{u}$ in (8) generalizes $c$ in (9) from constant functions to piecewise constant functions, so $\bar{u}$ in (14) generalizes the harmonic vector fields from $\mathcal{H}$ in (12) to piecewise harmonic vector fields. The second component of (14) appears to be a plausible definition of "motion texture".

\section{Denoising of Image Flows}

We take a closer look to the analogy between the ROF-model and our generalization to vector fields. Based on the definition (15), we propose to minimize the convex functional

$$
\frac{1}{2}\|u-d\|_{\Omega}^{2}+\lambda R(u)
$$

as a natural generalization of the ROF-model (1) to vector-valued data, where the regularizer $R$ is given by

$$
R(u)=\sup _{v \in S_{1}}\langle v, u\rangle_{\Omega} .
$$

Assuming $u$ to be sufficiently smooth, and taking into account the representation (15), in particular that the functions $\psi, \phi$ vanish at $\partial \Omega$, we can rewrite the regularizing term (17) as

$$
\begin{aligned}
R(u)=\sup _{v \in S_{1}}\langle v, u\rangle_{\Omega} & =\sup _{\|(\psi, \phi)\|_{\infty} \leq 1}\left\langle\nabla \psi+\nabla^{\perp} \phi, u\right\rangle_{\Omega} \\
& =\sup _{\|(\psi, \phi)\|_{\infty} \leq 1}\left\{-\langle\psi, \operatorname{div} u\rangle_{\Omega}+\langle\phi, \operatorname{curl} u\rangle_{\Omega}\right\} \\
& =\int_{\Omega} \sqrt{(\operatorname{div} u)^{2}+(\operatorname{curl} u)^{2}} d x
\end{aligned}
$$


and obtain (4). Since $R$ is positively homogeneous, $\lambda R(u)$ equals $\sup _{v \in S_{\lambda}}\langle v, u\rangle_{\Omega}$. Hence, minimizing (16) reads

$$
\inf _{u} \sup _{v \in S_{\lambda}}\left\{\frac{1}{2}\|u-d\|_{\Omega}^{2}+\langle v, u\rangle_{\Omega}\right\} .
$$

Exchanging inf and sup yields the convex decomposition

$$
d=u+v
$$

and the dual convex problem of (16)

$$
\inf _{v \in S_{\lambda}}\|v-d\|_{\Omega}^{2}
$$

In summary, the solution $\bar{u}$ of (16) is just given by (14). Let us point out again the similarities of our approach (16) for denoising vector fields with the ROF-model (1) for denoising scalar-valued functions, based on the convex Hodge decomposition introduced in the previous section. The regularizer $R$ in (17) is defined as support function of a convex set, as is the total variation measure TV in (6). Likewise, the dual convex problem (19) characterizes the non-smooth, oscillating component ("motion texture") of a given vector field $d$ as a projection onto a convex set, as does the ROF-model for scalar-valued functions in (7).

\section{Motion Boundaries}

Next we show by an heuristic example that for piecewise harmonic flows $u$, i.e., with vanishing divergence and curl a.e., the measure $R$ in (17) only contributes at motion boundaries.

Indeed, let us consider a partition $\bar{\Omega}=\left(\overline{\Omega_{1}} \cup \overline{\Omega_{2}}\right) \backslash \partial\left(\Omega_{1}, \Omega_{2}\right)$ with a smooth interior boundary $\partial\left(\Omega_{1}, \Omega_{2}\right)=\overline{\Omega_{1}} \cap \overline{\Omega_{2}}$, and a piecewise harmonic flow

$$
u=\left.u\right|_{\Omega_{1}}+\left.u\right|_{\Omega_{2}} \in \mathcal{H}
$$

that is discontinuous along $\partial\left(\Omega_{1}, \Omega_{2}\right)$. Applying Green's formulas [16],

$$
\begin{aligned}
\langle u, \nabla \psi\rangle_{\Omega}+\langle\operatorname{div} u, \psi\rangle_{\Omega} & =\int_{\partial \Omega}\langle u, n\rangle \psi d s, \quad \forall u \in H(\operatorname{div} ; \Omega), \forall \psi \in H^{1}(\Omega), \\
\langle\operatorname{curl} u, \phi\rangle_{\Omega}-\left\langle u, \nabla^{\perp} \phi\right\rangle_{\Omega} & =\int_{\partial \Omega}\langle u, t\rangle \phi d s, \quad \forall u \in H(\operatorname{curl} ; \Omega), \forall \phi \in H^{1}(\Omega),
\end{aligned}
$$

we obtain by $(\psi, \phi) \in\left(H_{0}^{1}(\Omega)\right)^{2}$ and $\sqrt{\psi(x)^{2}+\phi(x)^{2}} \leq 1$ due to (15) and (13) that

$$
\begin{aligned}
R(u)= & \sup _{\|(\psi, \phi)\|_{\infty} \leq 1}\left\langle\nabla \psi+\nabla^{\perp} \phi, u\right\rangle_{\Omega} \\
= & \sup _{\|(\psi, \phi)\|_{\infty} \leq 1}\left\{-\langle\psi, \operatorname{div} u\rangle_{\Omega_{1}}+\int_{\partial\left(\Omega_{1}, \Omega_{2}\right)}\left\langle u_{1}-u_{2}, n\right\rangle \psi d s\right. \\
& \left.\quad+\langle\phi, \operatorname{curl} u\rangle_{\Omega_{2}}-\int_{\partial\left(\Omega_{1}, \Omega_{2}\right)}\left\langle u_{1}-u_{2}, t\right\rangle \phi d s\right\}, \\
= & \int_{\partial\left(\Omega_{1}, \Omega_{2}\right)}\left\|u_{1}-u_{2}\right\| d s
\end{aligned}
$$


This generalizes in a plausible way the regularization term

$$
\left(u_{1}-u_{2}\right) \text { length }\left(\partial\left(\Omega_{1}, \Omega_{2}\right)\right)
$$

of the Mumford-Shah functional from piecewise-constant functions [6] to piecewise-harmonic vector fields.

\section{Algorithm}

In this section, we confine ourselves to deriving a simple iterative algorithm for solving the problem (19). The derivation of dedicated, more efficient algorithms, as was successfully done for the ROF-model $[18,19,20]$, is beyond the scope of this paper.

Throughout this section, we consider the discretized problem (19). The quantities $\psi, \phi, d$ are vectors of appropriate dimension, and $\nabla, \nabla^{\perp}$, div, curl, $\Delta$ are corresponding matrices preserving the identities fulfilled by the continuous operators, as detailed in [11]. Further, $\|\cdot\|_{\Omega}$ becomes an Euclidean inner product.

Proposition 7.1. For $0<\tau<2 /\|\Delta\|_{2}$, the sequence $\left\{\left(\psi^{(k)}, \phi^{(k)}\right)\right\}_{k \in \mathbb{N}}$ produced by the algorithm

$$
\begin{aligned}
&\left(\begin{array}{l}
\psi^{\left(k+\frac{1}{2}\right)} \\
\phi^{\left(k+\frac{1}{2}\right)}
\end{array}\right):=\left(\begin{array}{l}
\psi^{(k)} \\
\phi^{(k)}
\end{array}\right)+\tau\left(\begin{array}{c}
\Delta \psi^{(k)}-\operatorname{div} d \\
\Delta \phi^{(k)}+\operatorname{curl} d
\end{array}\right), \\
&\left(\begin{array}{c}
\psi^{(k+1)} \\
\phi^{(k+1)}
\end{array}\right):=\frac{\lambda}{\max \left\{\lambda,\left\|\left(\begin{array}{l}
\psi^{\left(k+\frac{1}{2}\right)} \\
\phi^{\left(k+\frac{1}{2}\right)}
\end{array}\right)\right\|_{2}\right\}}\left(\begin{array}{l}
\psi^{\left(k+\frac{1}{2}\right)} \\
\phi^{\left(k+\frac{1}{2}\right)}
\end{array}\right),
\end{aligned}
$$

converges for any initiatization $\left(\psi^{(0)}, \phi^{(0)}\right)$ to the minimizer $\bar{v}=\nabla \bar{\psi}+\nabla^{\perp} \bar{\phi}$ of (19).

Note that $\|\Delta\|_{2} \leq 8$, where the bound becomes sharp if the image size goes to infinity. The above algorithm was proposed without convergence proof for solving the ROF problem for scalar-valued images by Chambolle in [21]. The same author suggested another algorithm in [15].

Proof. In view of (15), we rewrite (19) as

$$
\inf _{\psi, \phi}\left\{F_{1}\left(\left(\begin{array}{c}
\psi \\
\phi
\end{array}\right)\right)+F_{2}\left(\left(\begin{array}{c}
\psi \\
\phi
\end{array}\right)\right)\right\}=\inf _{\psi, \phi}\left\{\left\|\nabla \psi+\nabla^{\perp} \phi-d\right\|_{\Omega}^{2}+\delta_{\mathbb{B}_{\infty ; \lambda}}\left(\left(\begin{array}{l}
\psi \\
\phi
\end{array}\right)\right)\right\},
$$

where $\delta_{\mathbb{B}_{\infty ; \lambda}}$ denotes the indicator function of $\mathbb{B}_{\infty ; \lambda}:=\left\{(\psi, \phi)^{\mathrm{T}}: \sqrt{\psi^{2}+\phi^{2}} \leq \lambda\right\}$ and the square and square root of vectors is meant componentwise. By virtue of (20) and orthogonality of $\nabla \psi$ and $\nabla^{\perp} \phi$, we obtain the optimality condition [22]

$$
\begin{aligned}
0 & \in \nabla F_{1}\left(\left(\begin{array}{c}
\bar{\psi} \\
\bar{\phi}
\end{array}\right)\right)+\partial F_{2}\left(\left(\begin{array}{c}
\bar{\psi} \\
\bar{\phi}
\end{array}\right)\right) \\
& =\left(\begin{array}{cc}
-\Delta & 0 \\
0 & -\Delta
\end{array}\right)\left(\begin{array}{c}
\bar{\psi} \\
\bar{\phi}
\end{array}\right)+\left(\begin{array}{c}
\operatorname{div} d \\
-\operatorname{curl} d
\end{array}\right)+\mathcal{N}_{\mathbb{B}_{\infty ; \lambda}}\left(\left(\begin{array}{c}
\bar{\psi} \\
\bar{\phi}
\end{array}\right)\right),
\end{aligned}
$$


where $\mathcal{N}_{\mathbb{B}_{\infty ; \lambda}}$ denotes the normal cone to the convex set $\mathbb{B}_{\infty ; \lambda}$. This inclusion leads for a positive constant $\tau>0$ to the fixed point equation

$$
\begin{aligned}
\left(I-\tau \nabla F_{1}\right)\left(\left(\begin{array}{c}
\bar{\psi} \\
\bar{\phi}
\end{array}\right)\right) & \in\left(I+\tau \partial F_{2}\right)\left(\left(\begin{array}{c}
\bar{\psi} \\
\bar{\phi}
\end{array}\right)\right) \\
\left(\begin{array}{c}
\bar{\psi} \\
\bar{\phi}
\end{array}\right) & =\left(I+\tau \partial F_{2}\right)^{-1}\left(I-\tau \nabla F_{1}\right)\left(\left(\begin{array}{c}
\bar{\psi} \\
\bar{\phi}
\end{array}\right)\right) \\
& =\prod_{\mathbb{B}_{\infty ; \lambda}}\left(\left(\begin{array}{c}
\bar{\psi} \\
\bar{\phi}
\end{array}\right)+\tau\left[\left(\begin{array}{cc}
\Delta & 0 \\
0 & \Delta
\end{array}\right)\left(\begin{array}{c}
\bar{\psi} \\
\bar{\phi}
\end{array}\right)-\left(\begin{array}{c}
\operatorname{div} d \\
-\operatorname{curl} d
\end{array}\right)\right]\right) .
\end{aligned}
$$

The corresponding Picard iteration (21), (22) is known as forward-backward splitting algorithm, see, e.g., [23]. Since the functional (23) is coercive there exists a minimizer. Further, $F_{2}$ is proper, convex and closed so that it remains by $[24,25]$ to show that $\nabla F_{1}$ is Lipschtz continuous with constant $<2 / \tau$ which is obviously the case if $\tau<2 /\|\Delta\|_{2}$.

\section{Experiments}

Figures 2 - 9 show results of various experiments concerning flow structure-texture decomposition, flow denoising, and the preservation of motion boundaries in order to substantiate the reasoning above. Detailed comments are given in the respective figure captions.

Comparing the performance of the standard TV-term (3) with our alternative term (17), resp. (4), the later shows the following advances:

- it leads to a flow decomposition $d=\bar{u}+\bar{v}$ with a richer structural component $\bar{u}$,

- it provides a plausible definition of motion texture $\bar{v}$ dependent on the scale parameter $\lambda$,

- it separates more accurately motion texture and noise from flow structure,

- and it better preserves motion boundaries.

\section{Conclusion}

Our work enlarges the class of convex variational approaches that can be used to denoise and separate vector-valued data. Our results elucidate a further mathematical setting where to some extent decisions (separation and segmentation) can be done just by convex programming, that is globally optimal.

In future work, we will continue this line of research and investigate more general variational approaches as well as related applications. Concerning the latter, an obvious class 
of data discussed already in the introduction above, are directional vector fields. Given a directional vector field $d$ with $\|d(x)\|_{2}=1$ a.e., we suggest the minimization problem

$$
\inf _{\|u\| \leq 1}\left\{-\langle d, u\rangle_{\Omega}+\lambda R(u)\right\}
$$

with $R$ from (17). This functional is convex. Does the solution satisfy $\|u(x)\|_{2}=1$ for any value $\lambda>0$ ? We will address this question in a subsequent paper and report here a preliminary numerical example only.
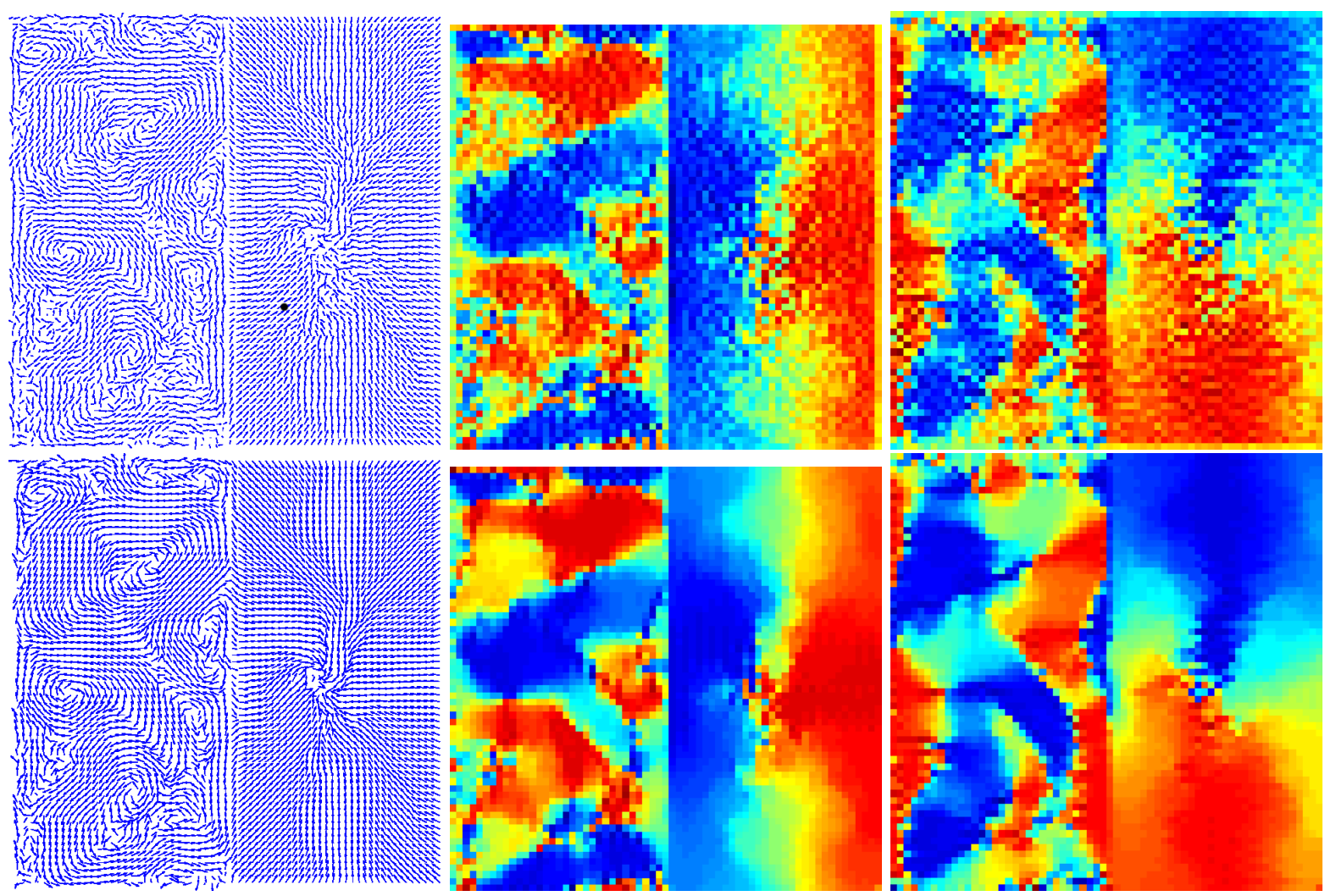

Figure 1: Top row: A directional flow field $d,\|d(x)\|=1$ is shown on the left, and its color-coded components $d_{1}, d_{2}$ in the center and on the right, respectively. Bottom row: The plots corresponding to the minimizer of (25), computed with the parameter value $\lambda=0.2$. Noise has been effectively removed while preserving $\|d(x)\|=1$ almost everywhere.

\section{References}

[1] L. Rudin, S. Osher, and E. Fatemi. Nonlinear total variation based noise removal algorithms. Physica D, 60:259-268, 1992.

[2] Y. Meyer. Oscillating Patterns in Image Processing and Nonlinear Evolution Equations, volume 22 of Univ. Lect. Series. Amer. Math. Soc., 2001. 
[3] J.-F. Aujol, G. Gilboa, T. Chan, and S. Osher. Structure-texture image decomposition - modeling, algorithms, and parameter selection. Int. J. Comp. Vision, 67(1):111-136, 2006.

[4] W.P. Ziemer. Weakly Differentiable Functions. Springer, 1989.

[5] T.F. Chan and J. Shen. Mathematical models for local non-texture inpaintings. SIAM J. Appl. Math., 61(4):1019-1043, 2001.

[6] D. Mumford and J. Shah. Optimal approximations by piecewise smooth functions and associated variational problems. Comm. Pure Appl. Math., 42:577-685, 1989.

[7] T.F. Chan and L.A. Vese. Active contours without edges. IEEE Trans. Image Proc., 10(2):266-277, 2001.

[8] T.F. Chan, S. Esedoglu, and M. Nikolova. Algorithms for finding global minimizers of image segmentation and denoising models. SIAM J. Appl. Math., 66(5):1632-1648, 2006.

[9] X. Bresson, S. Esedoglu, P. Vandergheynst, J.-P. Thiran, and S. Osher. Fast global minimization of the active contour/snake model. JMIV, 28(2):151-167, 2007.

[10] T.F. Chan and J. Shen. Image Processing and Analysis. Cambr. Univ. Press, 2005.

[11] J. Yuan, C. Schnörr, and G. Steidl. Simultaneous optical flow estimation and decomposition. SIAM J. Scientific Computing, 29(6):2283-2304, 2007.

[12] L. Vese and S. Osher. Numerical methods for $p$ - harmonic flows and applications to image processing. SIAM J. Numer. Anal., 40(6):2085-2104, 2002.

[13] O.M. Lysaker, S.J. Osher, and X.-C. Tai. Noise removal using smoothed normals and surface fitting. IEEE Trans. Image Proc., 13(10):1345-1357, 2004.

[14] D. Goldfarb, Z. Wen, and W. Yin. A curvilinear search method for p-harmonic flows on spheres. Technical Report CAAM TR08-02, Dept. Comp. Appl. Math., Rice University, 2007.

[15] A. Chambolle. An algorithm for total variation minimization and applications. J. Math. Imag. Vision, 20:89-97, 2004.

[16] V. Girault and P.-A. Raviart. Finite Element Methods for Navier-Stokes Equations. Springer, 1986.

[17] R. Dautray and J.-L. Lions. Mathematical Analysis and Numerical Methods for Science and Technology, volume 3. Springer, 2000. 
[18] T. Chan, G.H. Golub, and P. Mulet. A nonlinear primal-dual method for total variationbased image restoration. SIAM J. Sci. Comput., 20(6):1964-1977, 1999.

[19] K. Chen and X.-C. Tai. A nonlinear multigrid method for total variation minimization from image restoration. J. Sci. Comput., 33:115-138, 2007.

[20] D. Goldfarb and W. Yin. Second order cone programming methods for total variationbased image restoration. SIAM J. Scientific Computing, 2(27):622-645, 2005.

[21] A. Chambolle. Total variation minimization and a class of binary MRF models. In Anand Rangarajan, Baba C. Vemuri, and Alan L. Yuille, editors, Energy Minimization Methods in Computer Vision and Pattern Recognition, EMMCVPR, volume 3757 of Lecture Notes in Computer Science. Springer, 2005.

[22] R.T. Rockafellar and R.J.-B. Wets. Variational Analysis. Springer, 2004.

[23] P. L. Lions and B. Mercier. Splitting algorithms for the sum of two linear operators. SIAM J.J. Numer. Anal., 16:964-979, 1979.

[24] P. Tseng. Applications of a splitting algorithm to decomposition in convex programming and variational inequalities. SIAM J. Control Opt., 29(1):119-138, 1991.

[25] P. L. Combettes and V. R. Wajs. Signal recovery by proximal forward-backward splitting. Multiscale Modeling and Simulation, 4:1168-1200, 2005. 

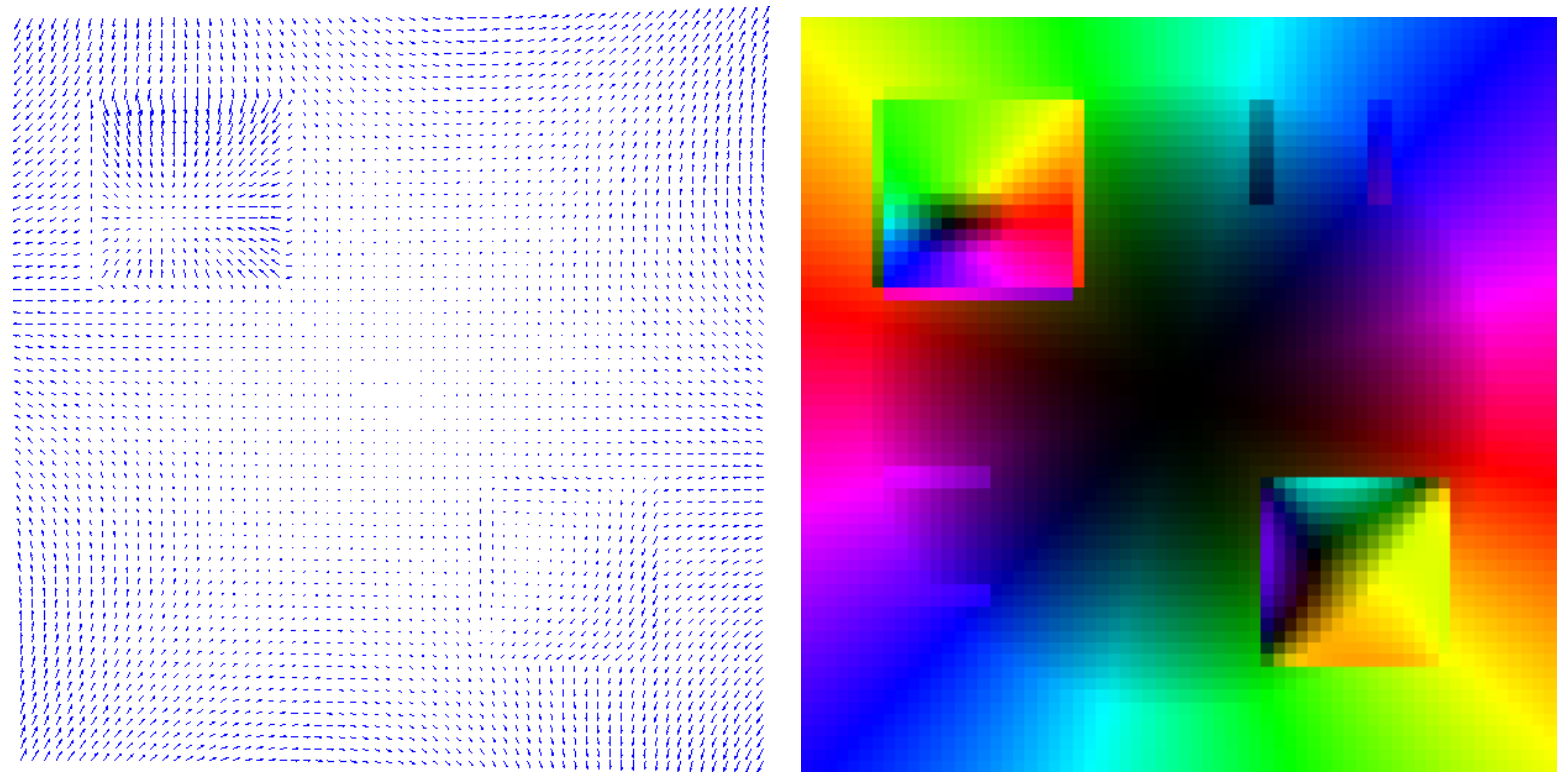

Figure 2: Vector field comprising 7 components (left: vector plot; right: colour plot of vector directions): a smooth harmonic flow in the background, two medium-scale non-harmonic flows in the upper-left and lower-right part, respectively, and four small-scale constant flows. 

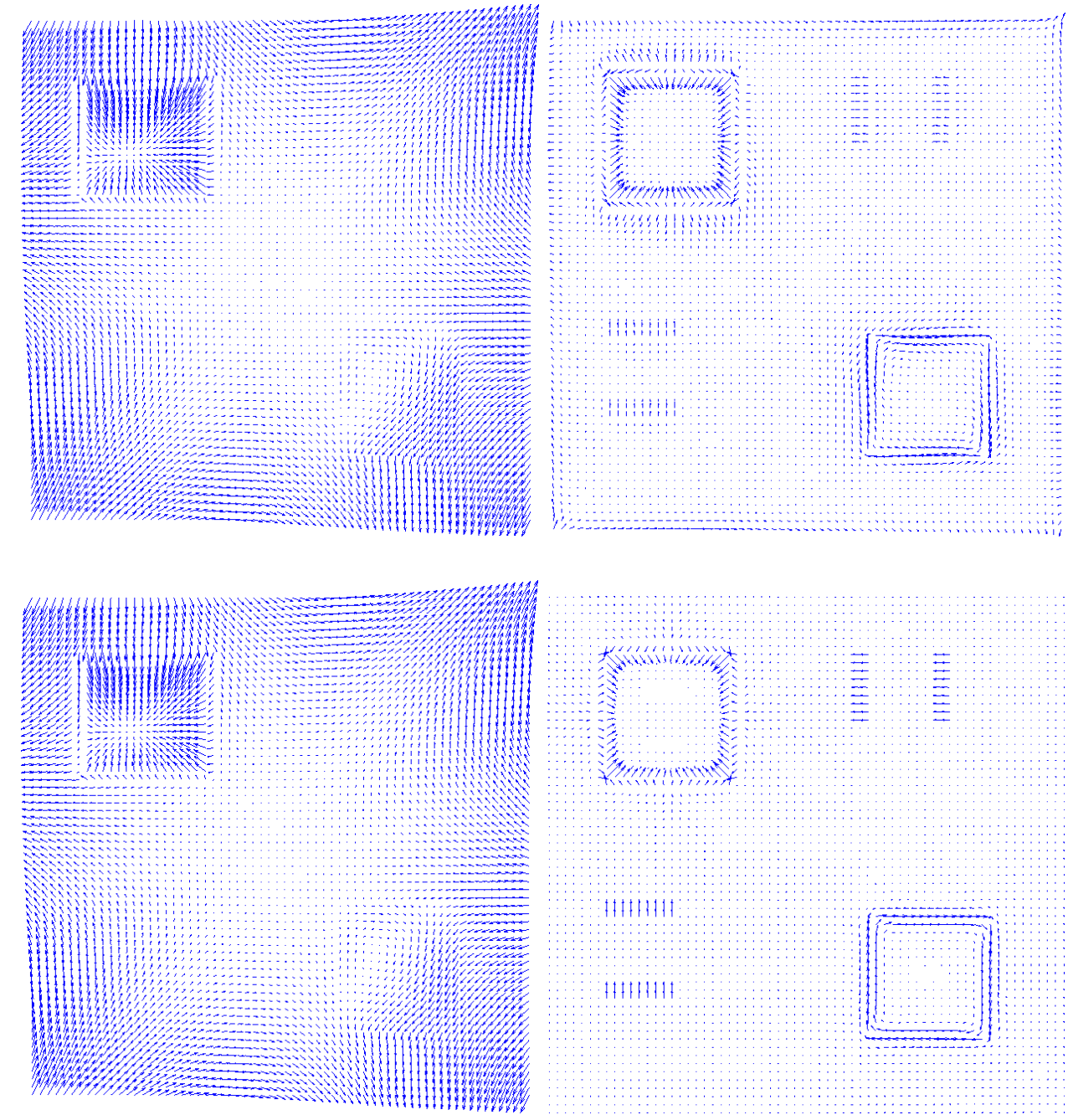

Figure 3: Decomposition $d=\bar{u}+\bar{v}$ of the flow $d$ shown in Fig. 2 for small $\lambda$ by the standard TV-term (3) (top row), and by the alternative definition (17) (bottom row). Left: structural part, Right: small-scale part. Due to the small value of $\lambda$, large- and medium-scale flows comprise the structure part $u$. Small-scale flow discontinuities, on the other hand, are more accurately determined by the alternative TV term (bottom right). 

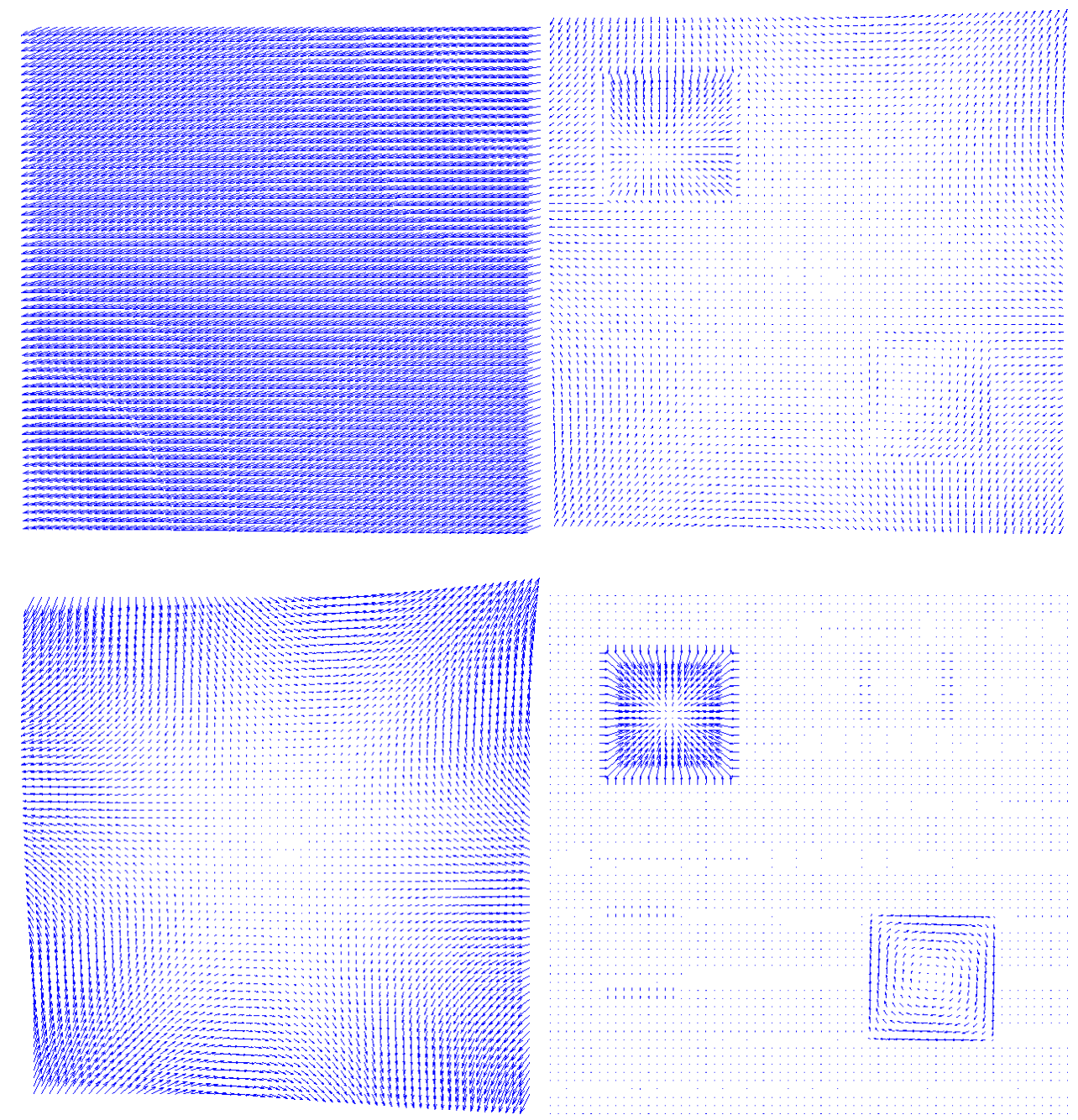

Figure 4: Decomposition $d=\bar{u}+\bar{v}$ of the flow $d$ shown in Fig. 2 for a large $\lambda$ by the standard TV-term (3) (top row), and by the alternative definition (17) (bottom row). Left: structural part, Right: small-scale part. Due to the large value of $\lambda$, the structure part $u$ returned by the standard term is an almost constant flow (top left), whereas the alternative term catches the large-scale harmonic flow in the background (bottom left). Accordingly, the standard term (3) recognizes almost all flow structure as "motion texture" (top right), whereas the alternative term (17) is able to separate the medium- and small-scale flows (bottom right). 

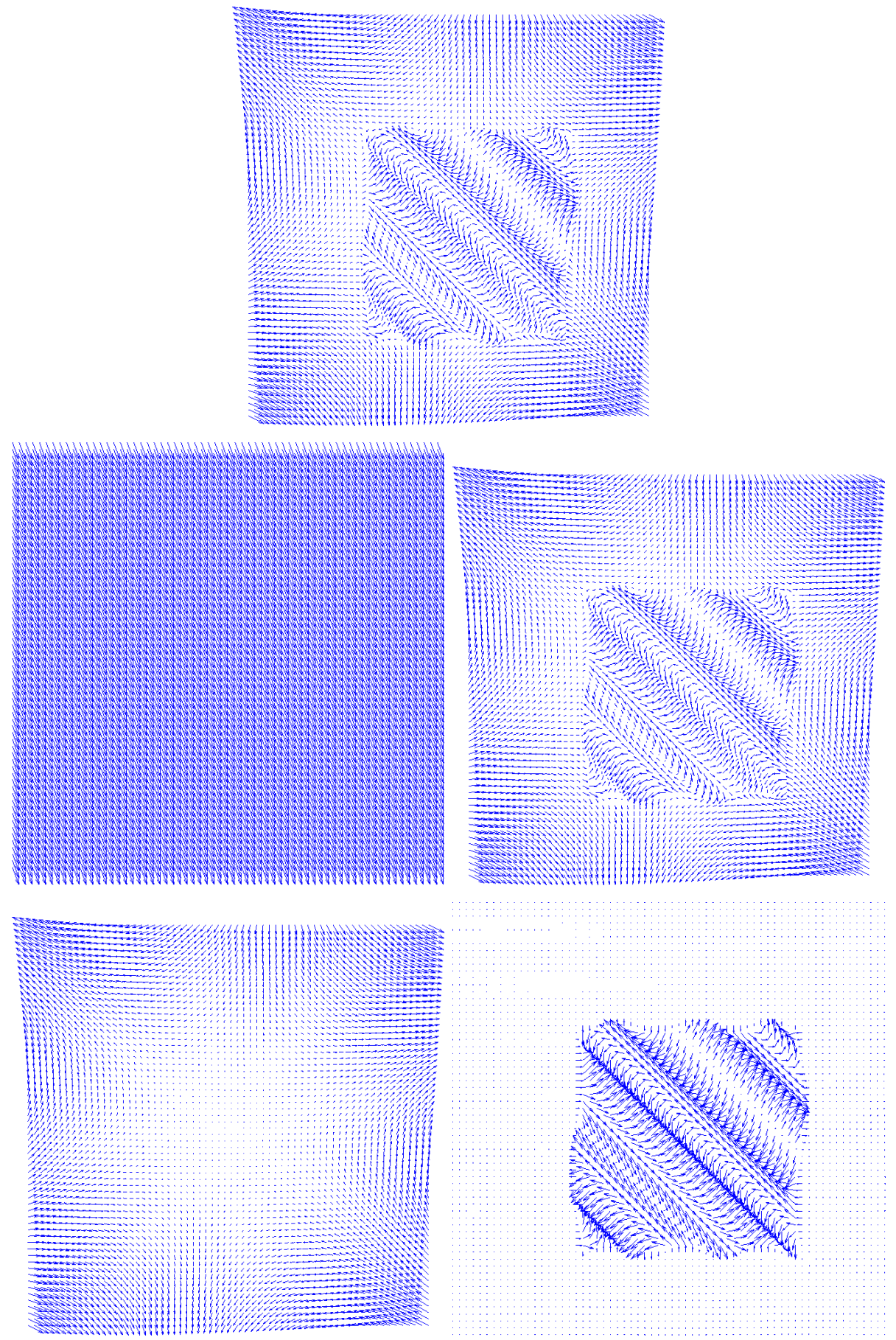

Figure 5: Decomposition $d=\bar{u}+\bar{v}$ of the flow $d$ shown in the top row, comprising a smooth harmonic flow and an arbitrary second flow (center) that varies at a smaller scale. As in the previous experiment the standard TV-term (3) (middle row) is not able to separate these two flows, whereas the alternative term (17) (bottom row) does so successfully. Notice that the parameter values were identical in both experiments, and that the motion texture separated by the novel term (bottom right) is not identical to the corresponding flow in the data (top row, middle part of the flow). This illustrates that motion texture $v$ is separated by an additive decomposition $d=\bar{u}+\bar{v}$. 

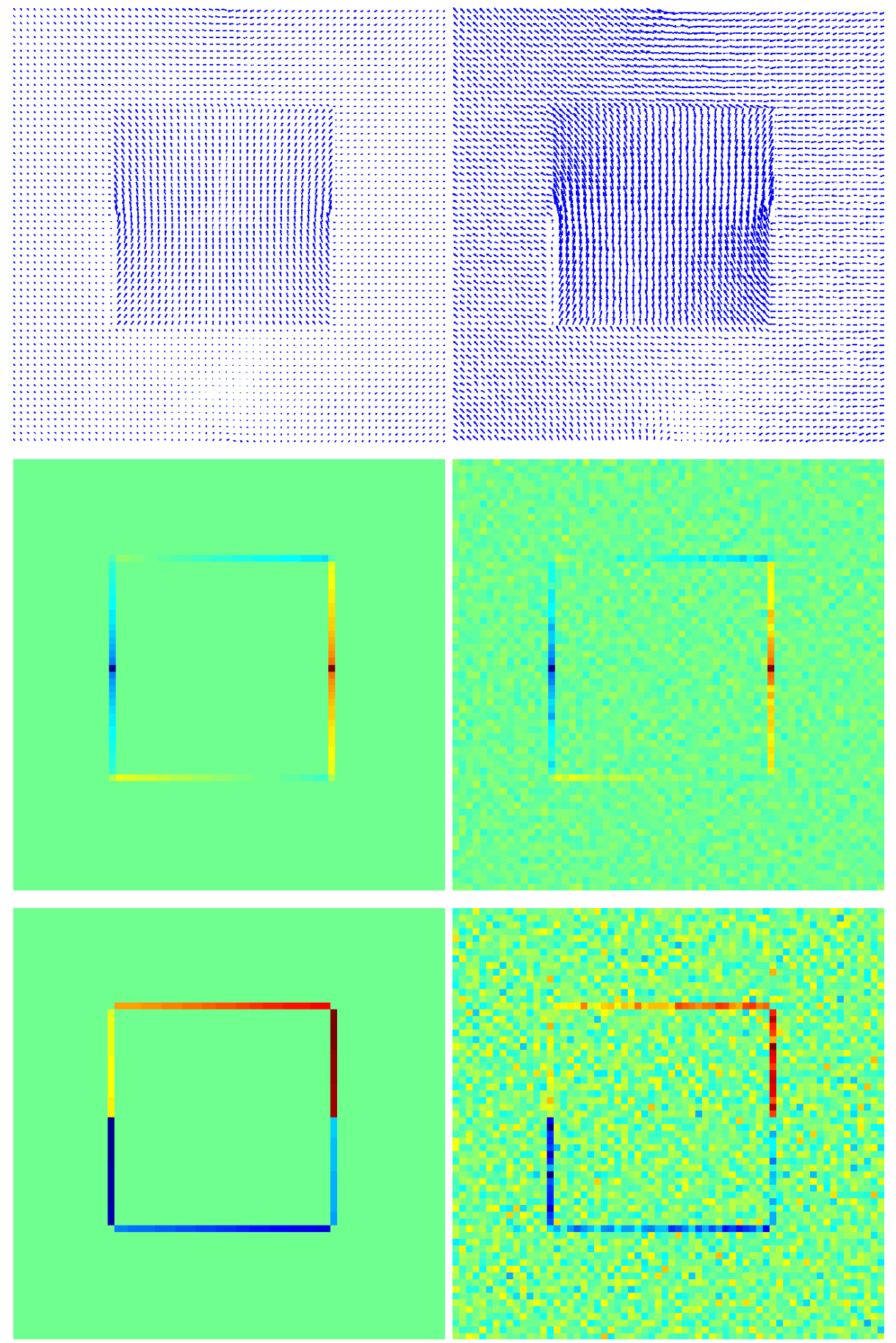

Figure 6: Data for the denoising experiment: Ground-truth flow $f$ (top left) and noisy input data $d$ (top right). Middle row: $\operatorname{curl}(f)$ and $\operatorname{curl}(d)$. Bottom row: $\operatorname{div}(f)$ and $\operatorname{div}(d)$. 

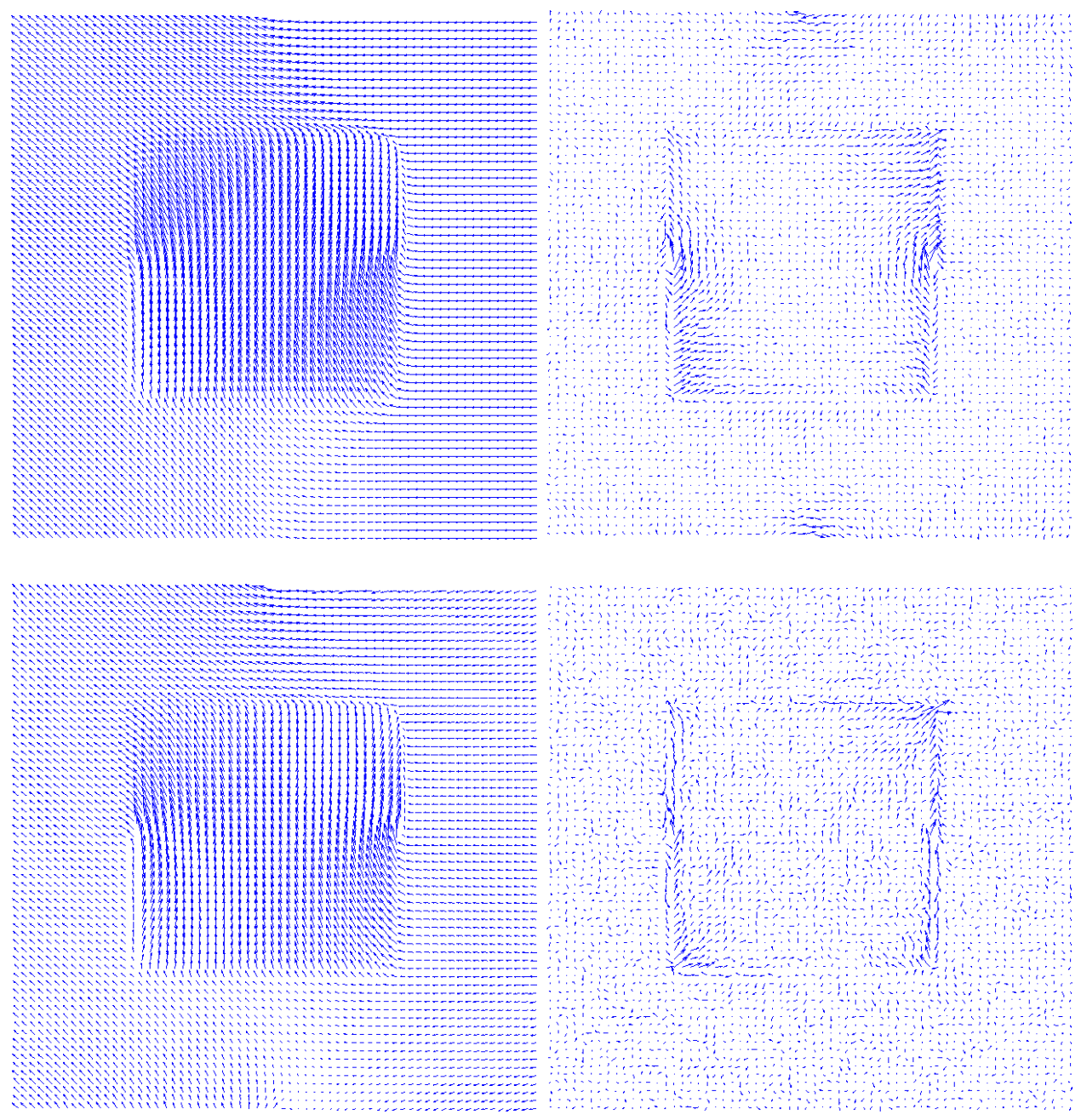

Figure 7: Decomposition $d=\bar{u}+\bar{v}$ of the noisy flow depicted in Fig. 6 using the standard TVterm (3) (top row) and the alternative term (17) (bottom row). The alternative term appears to separate more accurately signal from noise. Confer also Fig. 8 and Fig. 9 highlighting motion boundaries. 

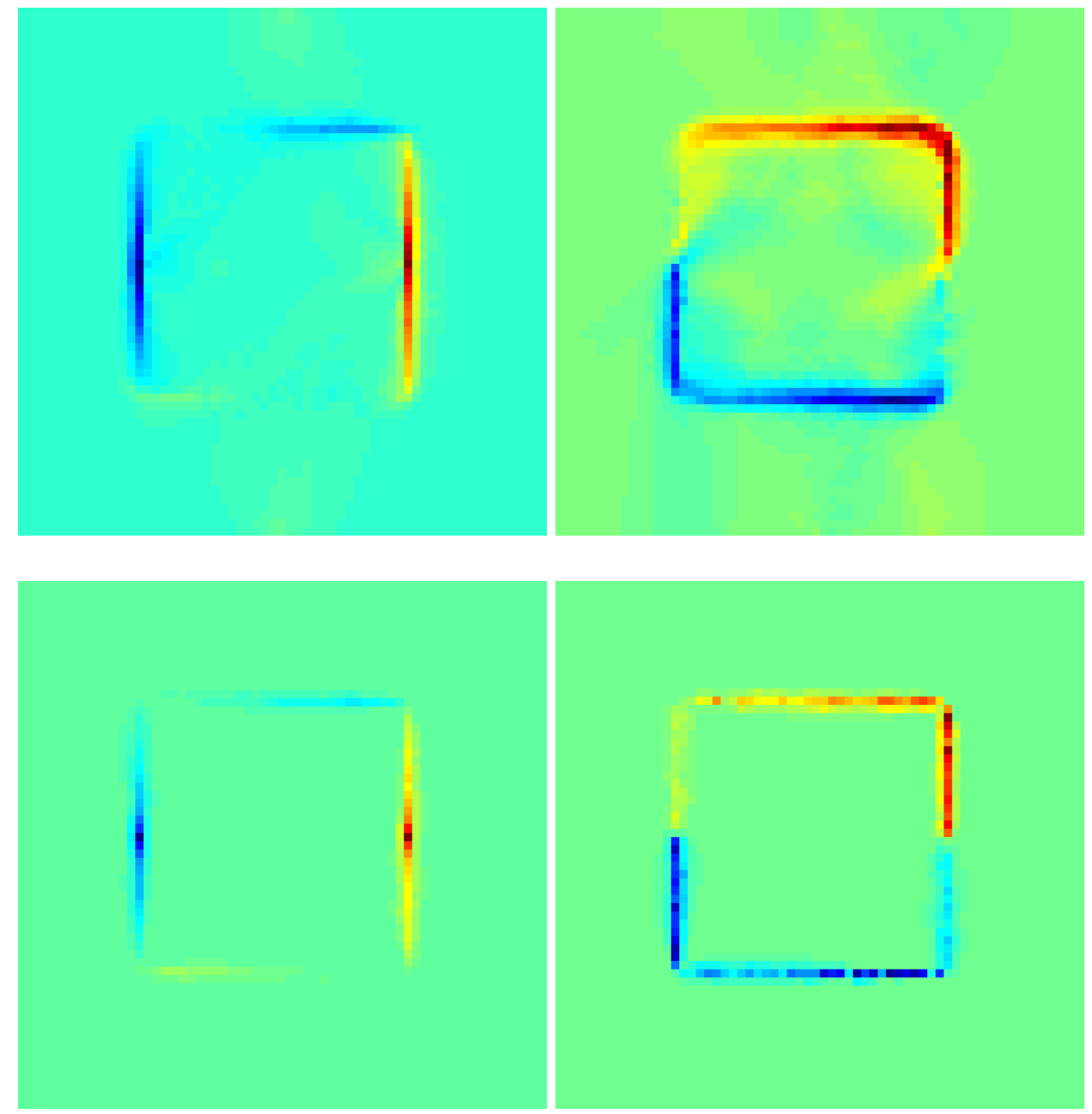

Figure 8: Decomposition $d=\bar{u}+\bar{v}$ of the noisy flow depicted in Fig. 6 using the standard TV-term (3) (top row) and the alternative term (17) (bottom row). The panels show $\operatorname{curl}(\bar{u})$ (left) and $\operatorname{div}(\bar{u})$ (right) corresponding to the flows depicted in Fig. 7 left. Motion boundaries are less blurred by the alternative term. 

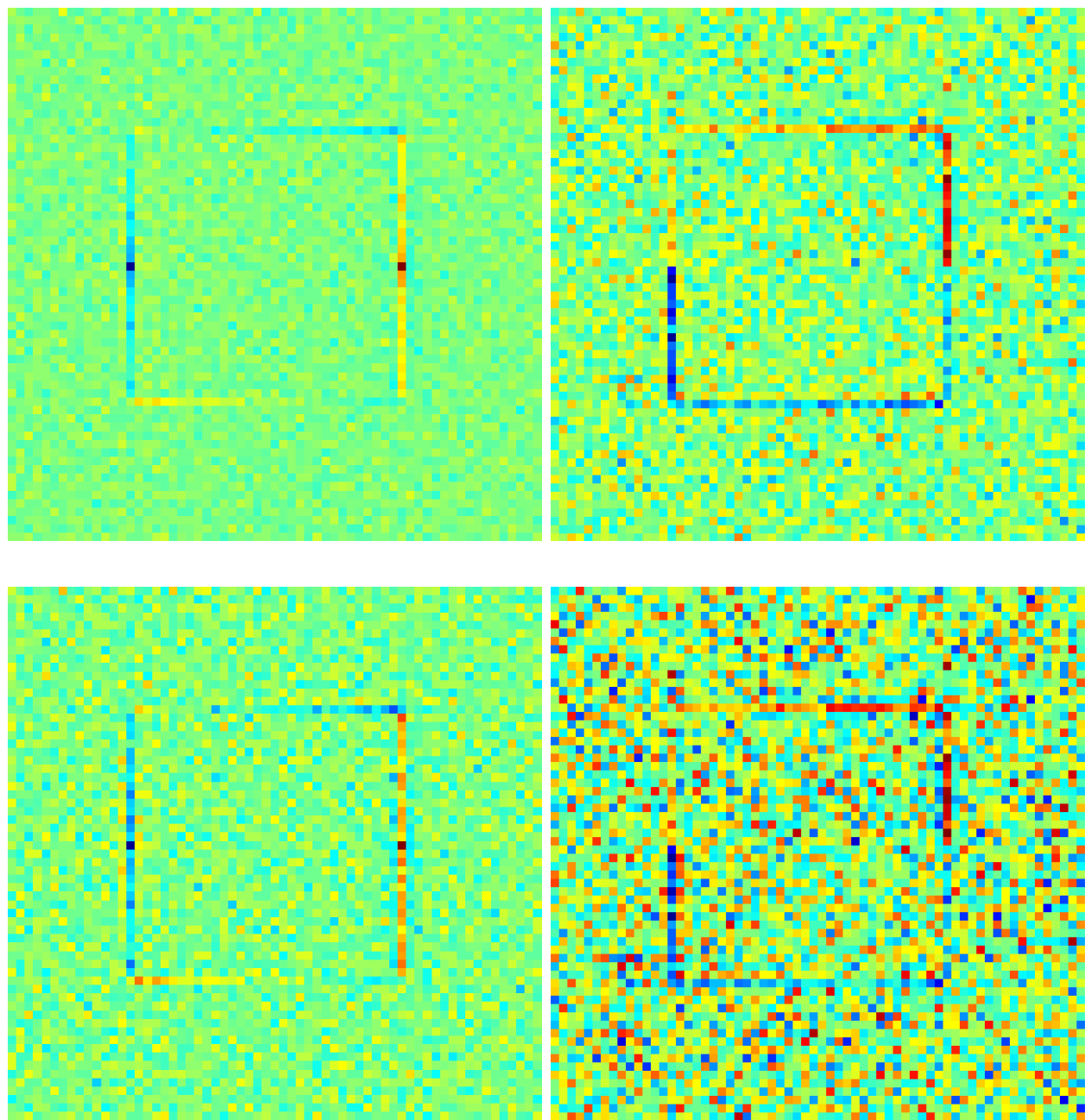

Figure 9: Decomposition $d=\bar{u}+\bar{v}$ of the flow depicted in Fig. 6 using the standard TV-term (3) (top row) and the alternative term (17) (bottom row). The panels show curl $(v)$ (left) and $\operatorname{div}(\bar{v})$ (right) corresponding to the flows depicted in Fig. 7 right. Noise is more accurately separated by the alternative term. 